\title{
Momentum Flux and Flux Divergence of Gravity Waves in Directional Shear Flows over Three-Dimensional Mountains
}

\author{
XIN XU \\ Key Laboratory of Mesoscale Severe Weather (Ministry of Education), and School of Atmospheric Sciences, Nanjing \\ University, Nanjing, China, and Center for Analysis and Prediction of Storms, Norman, Oklahoma \\ YUAN WANG \\ Key Laboratory of Mesoscale Severe Weather (Ministry of Education), and School of Atmospheric Sciences, \\ Nanjing University, Nanjing, China \\ Ming XUE \\ Center for Analysis and Prediction of Storms, and School of Meteorology, University of Oklahoma, Norman, \\ Oklahoma, and Key Laboratory of Mesoscale Severe Weather (Ministry of Education), and School of \\ Atmospheric Sciences, Nanjing University, Nanjing, China
}

(Manuscript received 9 January 2012, in final form 6 June 2012)

\begin{abstract}
Linear mountain wave theory is used to derive the general formulas of the gravity wave momentum flux (WMF) and its vertical divergence that develop in directionally sheared flows with constant vertical shear. Height variations of the WMF and its vertical divergence are studied for a circular bell-shaped mountain. The results show that the magnitude of the WMF decreases with height owing to variable critical-level height for different wave components. This leads to continuous-rather than abrupt-absorption of surface-forced gravity waves, and the rate of absorption is largely determined by the maximum turning angle of the wind with height. For flows turning substantially with height, the wave momentum is primarily trapped in the lower atmosphere. Otherwise, it can be transported to the upper levels. The vertical divergence of WMF is oriented perpendicularly to the right (left) of the mean flow that veers (backs) with height except at the surface, where it vanishes. First, the magnitude of the WMF divergence increases with height until reaching its peak value. Then, it decreases toward zero above that height. The altitude of peak WMF divergence is proportional to the surface wind speed and inversely proportional to the vertical wind shear magnitude, increasing as the maximum wind turning angle increases. The magnitude of the peak WMF divergence also increases with the maximum wind turning angle, but it in general decreases as the ambient flow Richardson number increases. Implications of the findings for treating mountain gravity waves in numerical models are discussed.
\end{abstract}

\section{Introduction}

Terrain-induced or mountain gravity waves are capable of vertically transporting horizontal momentum (Bretherton 1966). Once becoming unstable, these waves break up and deposit the momentum carried by them onto the ambient flow. Wave-mean flow interaction is enacted in association with the vertical divergence of wave momentum flux (WMF) (Fritts and Dunkerton 1984;

Corresponding author address: Yuan Wang, School of Atmospheric Sciences, Nanjing University, Nanjing 210093, China. E-mail: yuanasm@nju.edu.cn
Scinocca and Sutherland 2010). It has been recognized that gravity wave breaking plays an important role in balancing the momentum and thermal budgets of the atmospheric general circulation (Holton 1983; Palmer et al. 1986; McFarlane 1987).

Momentum transport by gravity waves in unidirectional flows has been studied extensively for several decades. Gravity waves can conserve their wave or perturbation momentum until encountering a preexisting or wave-induced critical level where the intrinsic wave frequency vanishes (Eliassen and Palm 1961; Booker and Bretherton 1967). For linear waves, the wave momentum is absorbed into a thin layer beneath the critical 
level, exerting a force on critical layer flow (Bretherton 1969; Lindzen 1981; Grubišić and Smolarkiewicz 1997). This force is known as gravity wave drag (GWD) (e.g., Kim et al. 2003), as it is directed in the opposite direction as basic wind except in the case of anisotropic obstacle (Phillips 1984; Teixeira and Miranda 2006).

For gravity waves generated in directional shear flows, however, the vertical wave momentum transport differs notably from the unidirectional flow case. The wave momentum is continuously absorbed by the mean flow over a range of height (Shutts and Gadian 1999) because a critical layer exists in the presence of directional wind shear (Shutts 1995). Doyle and Jiang (2006) studied a real mountain wave case in the southwestern Alps using observational data and numerical simulations, and confirmed the importance of directional wind shear on the upward propagation of mountain waves. Moreover, the vertical divergence of the WMF was shown to be perpendicular to the directional flow by Broad (1995). It is therefore more appropriate to call it a "lift" force (Smith 1979; Lott 1999), which acts to rotate the flow without changing its speed, physically different from the conventional GWD that acts to decelerate the mean flow (Fritts 1984).

Shutts (1995) studied the vertical distribution of the WMF divergence produced in directional shear flows using linear theory, but he only considered the situation of backing winds of limited rotation angles. The turning direction of the wind can have an important influence. Martin and Lott (2007) examined the synoptic response to the directional critical-level absorption of mountain waves triggered in cold and warm fronts. Their results show that a trough is produced over the mountain for cold fronts, whereas a ridge occurs in the case of warm fronts. Recently, Teixeira and Miranda (2009) derived expressions for the WMF and its vertical divergence for arbitrary directional shear flows varying relatively slowly with height, by using the Wentzel-KramersBrillouin (WKB) approximation. Height variations of the WMF were studied by them for some idealized wind profiles, but the WMF divergence for these profiles was not specifically addressed. This study extends the earlier studies by deriving general linear solutions of the mountain gravity waves, WMF, and WMF divergence for linear sheared flows of arbitrary degrees of rotation, and discusses the specific properties of the WMF divergence for several wind profiles with different shear magnitudes and degrees of rotation, and for waves over a circular bell-shaped mountain.

The rest of this paper is organized as follows. In section 2, the linearized wave equation is solved for flows varying linearly with height, using exact linear theory. In section 3, generic formulas are derived for the WMF and its vertical divergence. The formulas are then applied to an idealized three-dimensional (3D) circular bell-shaped mountain. Subsequently, height variations of the WMF and its vertical divergence are examined for six idealized wind profiles in section 4 . Further discussions and conclusions are given in sections 5 and 6 , respectively.

\section{Analytic solution of wave equations}

For a steady, inviscid, nonrotating, adiabatic, and hydrostatic Boussinesq flow, the linearized governing equations are of the form

$$
\begin{aligned}
U \frac{\partial u}{\partial x}+V \frac{\partial u}{\partial y}+U_{z} w & =-\frac{1}{\bar{\rho}} \frac{\partial p}{\partial x} \\
U \frac{\partial v}{\partial x}+V \frac{\partial v}{\partial y}+V_{z} w & =-\frac{1}{\bar{\rho}} \frac{\partial p}{\partial y} \\
\frac{1}{\bar{\rho}} \frac{\partial p}{\partial z} & =b \\
\frac{\partial u}{\partial x}+\frac{\partial v}{\partial y}+\frac{\partial w}{\partial z} & =0 \\
U \frac{\partial b}{\partial x}+V \frac{\partial b}{\partial y}+N^{2} w & =0
\end{aligned}
$$

Here, $\mathbf{V}(z)=[U(z), V(z)]$ is a horizontally homogeneous base-state wind with vertical wind shear $\mathbf{V}_{z}=$ $\left(U_{z}, V_{z}\right)$ (the subscript denotes a partial derivative with respect to the variable $), \mathbf{v}=(u, v, w)$ is perturbation velocity from base velocity $\mathbf{V}, \bar{\rho}$ is the constant reference density, and $p$ is the perturbation pressure from base-state pressure $p_{0} ; b=g \theta / \theta_{0}$ represents the net buoyancy force with $g$ being the gravitational acceleration, $\theta$ is the perturbation potential temperature from the base-state profile $\theta_{0}$, and $N^{2}=g d\left(\ln \theta_{0}\right) / d z$ is the Brunt-Väisälä frequency squared. Note that the base-state variables are dependent on height $z$, while all the perturbation variables are functions of the position vector $\mathbf{r}=(x, y, z)$.

Eliminating all perturbation variables except for vertical perturbation $w$ from Eqs. (1)-(5) leads to a single partial differential equation for $w$ as shown:

$$
\frac{\partial^{2} w}{\partial z^{2}}+\frac{N^{2} \nabla_{H}^{2}-D D_{z z}}{D^{2}} w=0,
$$

where $D=U \partial / \partial x+V \partial / \partial y$ denotes the horizontal advection by the base-state wind, with $\nabla_{H} \equiv(\partial / \partial x, \partial / \partial y)$ and $\nabla_{H}^{2} \equiv \partial^{2} / \partial x^{2}+\partial^{2} / \partial y^{2}$ being the horizontal gradient and Laplace operators, respectively. Substituting the two-dimensional (2D) Fourier transform pairs of the vertical velocity, namely, 
$w(x, y, z)=\int_{-\infty}^{+\infty} \int_{-\infty}^{+\infty} \hat{w}(k, l, z) \exp [i(k x+l y)] d k d l$,

$\hat{w}(k, l, z)=\frac{1}{4 \pi^{2}} \int_{-\infty}^{+\infty} \int_{-\infty}^{+\infty} w(x, y, z) \exp [-i(k x+l y)] d x d y$,

into Eq. (6) results in the well-known Taylor-Goldstein equation (Teixeira et al. 2004) as shown:

$$
\frac{\partial^{2} \hat{w}}{\partial z^{2}}+\frac{N^{2} K^{2}-\hat{D} \hat{D}}{\hat{D}^{2}} \hat{w}=0,
$$

where $\mathbf{K}=(k, l)$ is the horizontal wave vector, $K=$ $|\mathbf{K}|$, and $\hat{D}(z)=\mathbf{V}(z) \cdot \mathbf{K}$. For linear gravity waves, Eq. (9) is subjected to free-slip condition at the bottom boundary

$$
\hat{w}(k, l, 0)=i \hat{D}_{0} \hat{h}(k, l),
$$

where $\hat{D}_{0}=\mathbf{V}_{0} \cdot \mathbf{K}$ and $\hat{h}(k, l)$ is the 2D Fourier transform of terrain height $h(x, y)$. Moreover, it obeys the radiation condition aloft, so that only upward energy propagation is allowed as $z \rightarrow+\infty$ (Booker and Bretherton 1967).

In this study, an idealized wind profile varying linearly with height is adopted as shown:

$$
\mathbf{V}(z)=\left(U_{0}+U_{z} z, V_{0}+V_{z} z\right),
$$

where $\mathbf{V}_{0}=\left(U_{0}, V_{0}\right)$ is the base-state wind at the surface $(z=0)$. Using this linearly sheared wind, the curvature effect of the wind can be a priori excluded from Eq. (9) as $\mathbf{V}_{z z}=0$. For convenience of calculation, the following polar coordinates are introduced:

$$
\begin{aligned}
\mathbf{K} & =K(\cos \varphi, \sin \varphi), \\
\mathbf{V}(z) & =|\mathbf{V}(z)|[\cos \psi(z), \sin \psi(z)],
\end{aligned}
$$

where $\psi(z)$ is the azimuth of base-state wind at height $z$. By virtue of Eqs. (10)-(13), the analytic, physically meaningful solution of Eq. (9) can be obtained using the Frobenius method (Booker and Bretherton 1967; Grubišić and Smolarkiewicz 1997) as shown:

$$
\begin{aligned}
\hat{w}(K, \varphi, z)= & i \hat{D}_{0} \hat{h}(K, \varphi)\left|1-z / z_{c}\right|^{1 / 2+i \operatorname{sgn}\left[\hat{D}_{z}\left(z_{c}\right)\right] \mu(\varphi)} \\
& \times \exp \left(\pi \mathcal{H}\left(z-z_{c}\right)\left\{i \operatorname{sgn}\left[\hat{D}_{z}\left(z_{c}\right)\right] / 2-\mu(\varphi)\right\}\right),
\end{aligned}
$$

where

$$
\begin{gathered}
z_{c}(\varphi)=-\frac{\hat{D}_{0}}{\hat{D}_{z}}=-\frac{\left|\mathbf{V}_{0}\right| \cos \left(\psi_{0}-\varphi\right)}{\left|\mathbf{V}_{z}\right| \cos \left(\chi_{0}-\varphi\right)}, \\
\mu(\varphi)=\sqrt{\mathrm{Ri} / \cos ^{2}\left(\chi_{0}-\varphi\right)-0.25} .
\end{gathered}
$$

In Eqs. (14)-(16), $z_{c}(\varphi)$ is the critical-level height corresponding to the wave vector oriented in direction $\varphi$, satisfying the general critical-level condition $\mathbf{V}\left(z_{c}\right) \cdot \mathbf{K}=0$; $\psi_{0}$ and $\chi_{0}$ represent the direction of $\mathbf{V}_{0}$ and $\mathbf{V}_{z}$, respectively; $\mathrm{Ri} \equiv N^{2} /\left|\mathbf{V}_{z}\right|^{2}$ is the ambient flow Richardson number and is greater than 0.25 for stably stratified flows; $\operatorname{sgn}(\cdot)$ is the sign function; and $\mathcal{H}(x)$ designates the Heaviside function, which is equal to zero and unity for negative and positive argument $x$, respectively.

The exponential term on the right-hand side of Eq. (14) represents the fraction of a wave component that can pass through its critical level, because $\mathcal{H}\left(z-z_{c}\right)$ is equal to unity above $z_{c}$ and zero below $z_{c}$. The attenuation of the wave amplitude is $\exp (-\pi \mu)$ on passing through the critical level. Since transmitted waves have to cross the critical level again from above to influence the flow below, the double-attenuated waves can retain at most about 0.04 of their initial amplitude at $\mathrm{Ri}$ as low as 0.5 (Teixeira et al. 2005). Accordingly, wave transmission through the critical level is omitted by dropping the exponential term in Eq. (14), so that it is simplified to

$$
\hat{w}(K, \varphi, z)=i \hat{D}_{0} \hat{h}(K, \varphi)\left|1-z / z_{c}\right|^{1 / 2+i \operatorname{sgn}\left[\hat{D}_{z}\left(z_{c}\right)\right] \mu(\varphi)} .
$$

\section{Formulas of wave momentum flux and its vertical divergence}

\section{a. General formulas}

According to Lin (2007), the negative of the vertical flux of horizontal wave momentum at the surface is equal to the pressure force exerted on the mountain by the airflow [see Eqs. (5.1.24) and (5.1.25) of Lin (2007)]. Following the convention, a minus sign is included in the definition of the WMF, as in Teixeira and Miranda (2009), to make it generally positive. Accordingly, the WMF at the surface, written in the Fourier space [see Eq. (16) of Teixeira et al. (2004)], is

$$
\mathbf{F}_{0}=4 \pi^{2} i \int_{-\infty}^{+\infty} \int_{-\infty}^{+\infty}(\cos \varphi, \sin \varphi) K^{2} \hat{p} *(z=0) \hat{h}(K, \varphi) d K d \varphi,
$$

where the asterisk denotes a complex conjugate.

The pressure perturbation can be readily obtained by eliminating $u$ and $v$ from Eqs. (1), (2), and (4) so that

$$
\hat{p}=i \frac{\bar{\rho}}{K^{2}}\left(\hat{D}_{z}-\hat{D} \frac{\partial}{\partial z}\right) \hat{w} .
$$

Substituting the above equation and Eqs. (15)-(17) into Eq. (18) yields 
(a)

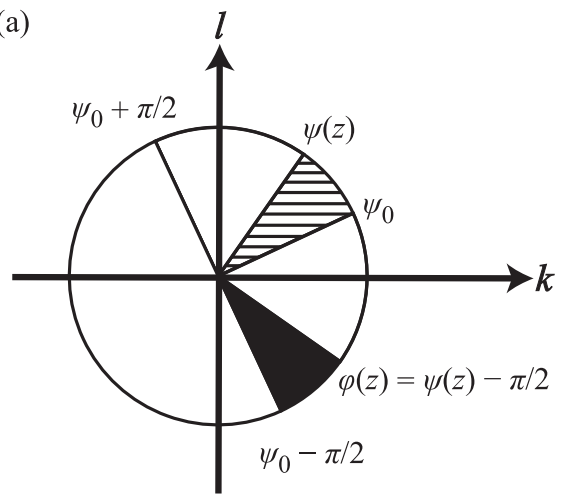

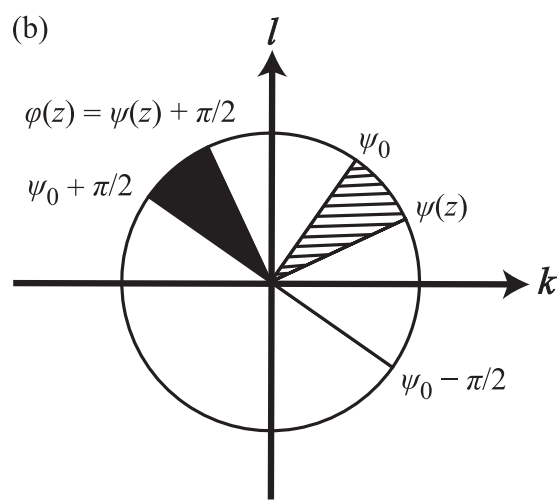

(b)

FIG. 1. Schematic diagram for continuous critical-level filtering of WMF with height, for (a) backing winds and (b) veering winds. From the surface to height $z$, base-state winds rotate from the azimuth of $\psi_{0}$ to $\psi(z)$ (hatched region); the wave components with the wavenumber vector in the blackened sector are filtered (their wave momentum fluxes vanish).

$$
\mathbf{F}_{0}=\left.8 \bar{\rho} N \pi^{2}\left|\mathbf{V}_{0}\right| \int_{0}^{\infty} \int_{0}^{\pi}(\cos \varphi, \sin \varphi) \cos \left(\varphi-\psi_{0}\right) \sqrt{1-\frac{\cos ^{2}\left(\chi_{0}-\varphi\right)}{4 \mathrm{Ri}}} \hat{h}(K, \varphi)\right|^{2} K^{2} d K d \varphi,
$$

where $\hat{w}(K, \varphi)=\hat{w}^{*}(K, \varphi \pm \pi)$ has been used.

As mentioned earlier, the WMF in directional shear flows varies with height. Figure 1 schematically shows the directional critical-level filtering of WMF. The WMF at the surface comprises the contribution of all wave components with wavenumber vectors in the azimuth range of $\left(\psi_{0}-\pi / 2, \psi_{0}+\pi / 2\right)$. [Note that wave components in the range of $\left(\psi_{0}+\pi / 2, \psi_{0}+3 \pi / 2\right)$ have a contribution equivalent to that of their conjugate.] When moving upward from surface to $z$, the basic wind turns with height from direction $\psi_{0}$ to $\psi(z)$ (as covered by the hatched sector in Fig. 1). The wave components with wavenumber vectors in the azimuth range of $\left(\psi_{0}-\pi / 2, \psi[z]-\pi / 2\right)$ (blackened sector in Fig. 1a), or $\left(\psi[z]+\pi / 2, \psi_{0}+\pi / 2\right)$ (blackened sector in Fig. 1b), depending on whether the wind backs or veers with height, encounter their own critical levels consecutively and hence are removed from the wave packet. In consequence, the remaining WMF at $z$ becomes

$$
\mathbf{F}(z)=8 \bar{\rho} N \pi^{2}\left|\mathbf{V}_{0}\right| \int_{0}^{\infty} \int_{\varphi_{L}}^{\varphi_{U}}(\cos \varphi, \sin \varphi) \cos \left(\varphi-\psi_{0}\right) \sqrt{1-\frac{\cos ^{2}\left(\chi_{0}-\varphi\right)}{4 \mathrm{Ri}}} \mid \hat{h}^{2} K^{2} d K d \varphi
$$

where $\varphi_{L}=\psi(z)-\pi / 2, \varphi_{U}=\psi_{0}+\pi / 2$ for backing winds and $\varphi_{L}=\psi_{0}-\pi / 2, \varphi_{U}=\psi(z)+\pi / 2$ for veering winds.

Differentiating Eq. (21) with respect to $z$ immediately gives the vertical divergence of the WMF as shown:

$$
\begin{aligned}
\mathbf{L}(z)= & \pm\left[\mathbf{k} \times \frac{\mathbf{V}(z)}{|\mathbf{V}(z)|}\right] 8 \bar{\rho} N^{2} \pi^{2}\left|\mathbf{V}_{0}\right| \\
& \times \frac{\sin \left|\psi(z)-\psi_{0}\right|}{|\mathbf{V}(z)|} \sqrt{1-\left\{1-\frac{\sin ^{2}\left[\chi_{0}-\psi(z)\right]}{2 \mathrm{Ri}}\right\}^{2}} \\
& \times \int_{0}^{\infty} K^{2}|\hat{h}[K, \psi(z)]|^{2} d K
\end{aligned}
$$

where $\mathbf{k}$ is the vertical unit vector, and the plus and minus signs apply to backing and veering winds, respectively. It is evident that $\mathbf{L}(z)$ is only nonzero above ground because $\sin \left|\psi(z)-\psi_{0}\right|$ is trivially zero at $z=0$. Additionally, $\mathbf{L}(z)$ is always perpendicular to base-state wind vector $\mathbf{V}(z)$. It is directed to the left of the wind that backs with height but to the right of veering wind. A schematic illustration of $\mathbf{L}(z)$ for linearly sheared backing and veering wind profiles is given in Fig. 2.

\section{b. WMF and its vertical divergence for a circular bell-shaped mountain}

In theoretical studies of terrain-generated gravity waves, three-dimensional circular bell-shaped mountains are commonly used as the forcing topography (Crapper 1959; Smith 1980; Shutts and Gadian 1999; Teixeira et al. 2004). It is defined by 
(a)

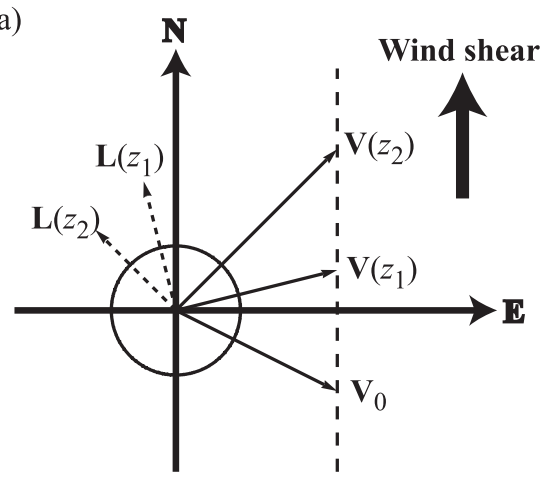

(b)

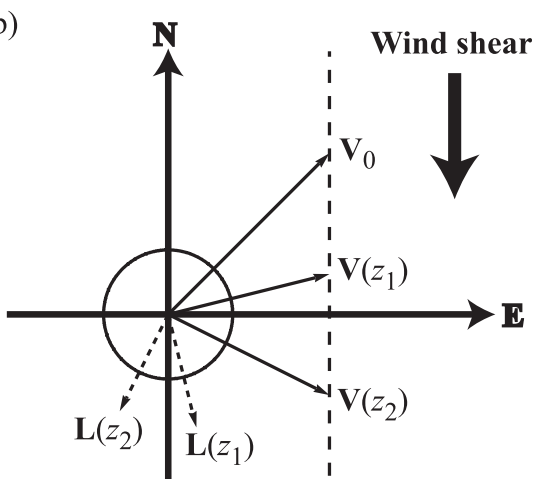

FIG. 2. Schematic diagram of the WMF divergence produced by (a) backing and (b) veering base-state horizontal winds over a circular bell-shaped mountain (illustrated by the solid circle). Thick arrow denotes the direction of the vertical wind shear. Variables $\mathbf{V}_{0}, \mathbf{V}\left(z_{1}\right)$, and $\mathbf{V}\left(z_{2}\right)$ represent the base-state winds at the heights of $z=0, z_{1}$, and $z_{2}$, respectively, while $\mathbf{L}\left(z_{1}\right)$ and $\mathbf{L}\left(z_{2}\right)$ designate the WMF divergence at $z=z_{1}$ and $z_{2}$, respectively; $\mathbf{L}\left(z_{1}\right)$ and $\mathbf{L}\left(z_{2}\right)$ are perpendicular to $\mathbf{V}\left(z_{1}\right)$ and $\mathbf{V}\left(z_{2}\right)$, respectively.

$$
h(x, y)=h_{0}\left[1+(x / a)^{2}+(y / a)^{2}\right]^{-3 / 2},
$$

where $h_{0}$ and $a$ are the mountain height and half-width, respectively. This circular mountain is isotropic, whose 2D Fourier transform is independent of azimuth $\varphi$; that is,
Substituting the above equation into Eqs. (21) and (22) yields

$$
\mathbf{F}(z)=0.5 \bar{\rho} N a h_{0}^{2}\left|\mathbf{V}_{0}\right| \int_{\varphi_{L}}^{\varphi_{U}}(\cos \varphi, \sin \varphi) \cos \left(\psi_{0}-\varphi\right) \sqrt{1-\cos ^{2}\left(\chi_{0}-\varphi\right) /(4 \mathrm{Ri})} d \varphi,
$$

$$
\begin{aligned}
\mathbf{L}(z)= & \pm\left[\mathbf{k} \times \frac{\mathbf{V}(z)}{|\mathbf{V}(z)|}\right] 0.5 \bar{\rho} N^{2} a h_{0}^{2}\left|\mathbf{V}_{0}\right| \\
& \times \frac{\sin \left|\psi(z)-\psi_{0}\right|}{|\mathbf{V}(z)|} \sqrt{1-\left\{1-\frac{\sin ^{2}\left[\chi_{0}-\psi(z)\right]}{2 \mathrm{Ri}}\right\}^{2}} .
\end{aligned}
$$

For unidirectional shear flows where $\chi_{0}= \pm \psi_{0}$, Eq. (25) reduces to the result of Grubišić and Smolarkiewicz (1997) as shown:

$\mathbf{F}_{\text {uni }}=0.5 \bar{\rho} N a h_{0}^{2} \mathbf{V}_{0} \int_{0}^{\pi} \cos ^{2} \varphi \sqrt{1-\cos ^{2} \varphi / 4 \mathrm{Ri}} d \varphi$,

which further reduces to that of Teixeira et al. (2004) as follows:

$$
\mathbf{F}_{\text {cons }}=0.25 \pi \bar{\rho} N a h_{0}^{2} \mathbf{V}_{0},
$$

for constant flows with $\mathbf{V}_{z}=0$ (i.e., Ri $=\infty$ ). Obviously, the WMFs in both Eqs. (27) and (28) are independent of height. Moreover, they are parallel to the base-state wind vector, which is ascribed not only to the isotropy of the circular mountain but also to the unidirectionality of the mean flow, as will be shown in section 4a. In the situation of directional shear wind, the WMF given by Eq. (25) is height dependent, thus giving rise to nonzero vertical divergence of WMF as given by Eq. (26). According to our knowledge, the above-mentioned general formulas have not been documented in meteorological literature before.

\section{WMF and its vertical divergence for various wind profiles}

In the preceding section, the general formulas for the WMF and its vertical divergence were derived and the special solution for a 3D circular bell-shaped mountain was obtained. This section will examine the properties of these formulas in more detail for specific wind profiles. Six wind profiles will be examined; they have the same surface wind speed of $\left|\mathbf{V}_{0}\right|=8 \mathrm{~m} \mathrm{~s}^{-1}$ but distinct surface wind direction and vertical wind shear (Table 1). Because the direction of the wind is asymptotic to that of the vertical wind shear as $z \rightarrow+\infty$, the maximum turning angle of the wind with height is given by 
TABLE 1. Parameters of six directional shear wind profiles with constant vertical wind shear magnitude $\left|\mathbf{V}_{z}\right|$. Symbols are defined in the text. Ri is obtained by supposing a constant Brunt-Väisälä frequency of $N^{2}=10^{-4} \mathrm{~s}^{-2}$.

\begin{tabular}{|c|c|c|c|c|c|c|c|}
\hline & $\left|\mathbf{V}_{0}\right|$ & $\left|\mathbf{V}_{z}\right|\left(\mathrm{s}^{-1}\right)$ & $\mathrm{Ri}$ & $\psi_{0}$ & $\chi_{0}$ & $\Phi\left(^{\circ}\right)$ & $\Phi_{8 \mathrm{~km}}\left({ }^{\circ}\right)$ \\
\hline W1 & \multirow{6}{*}{$8 \mathrm{~m} \mathrm{~s}^{-1}$} & $5 \times 10^{-3}$ & 4 & $\pi / 4$ & $\pi / 2$ & 45 & 37.9 \\
\hline W2 & & $5 \times 10^{-3}$ & 4 & 0 & $\pi / 2$ & 90 & 78.7 \\
\hline W3 & & $5 \times 10^{-3}$ & 4 & $-\pi / 4$ & $\pi / 2$ & 135 & 125.6 \\
\hline W4 & & $5 \times 10^{-3}$ & 4 & $\pi / 4$ & $-\pi / 2$ & 135 & 125.6 \\
\hline W5 & & $10^{-2}$ & 1 & $\pi / 4$ & $-\pi / 2$ & 135 & 128.7 \\
\hline W6 & & $\sqrt{2} \times 10^{-2}$ & 0.5 & $\pi / 4$ & $-\pi / 2$ & 135 & 130.6 \\
\hline
\end{tabular}

$$
\Phi=\left|\chi_{0}-\psi_{0}\right|
$$

Accordingly, the first three winds turn counterclockwise with height by $\Phi=45^{\circ}, 90^{\circ}$, and $135^{\circ}$, while the other three undergo an identical clockwise rotation of $\Phi=135^{\circ}$. Moreover, the vertical wind shear magnitudes are the same for the first four wind profiles, while the final two have shear magnitudes 2 and $2 \sqrt{2}$ times larger, respectively.

These ambient wind profiles along with $N^{2}=10^{-4} \mathrm{~s}^{-2}$ are substituted into Eqs. (25) and (26) to obtain the WMF and its vertical divergence. While Eq. (26) can be easily calculated, evaluating the nonelementary integral in Eq. (25) is much more difficult so we perform it numerically.

\section{a. Wave momentum flux}

Figures $3 \mathrm{a}$ and $3 \mathrm{~d}$ display the normalized WMFs as a function of height. The normalization is with respect to the WMF produced in a constant flow having the same surface speed, as given by Eq. (28). As expected, the magnitude of the WMF decreases with height (Shutts and Gadian 1999), in particular for those winds with a large asymptotic turning angle $\Phi$. For instance, while the WMF is decreased by only about $6.5 \%$ at $z=8 \mathrm{~km}$ for the wind W1 (dotted line in Fig. 3a and Table 1), wind $\mathrm{W} 3$ has a reduction of about $74 \%$ at the same height (solid line in Fig. 3a). The WMFs for the latter three winds undergo a similar decrease at $z=8 \mathrm{~km}$ (Fig. 3d), despite their distinctly different magnitudes of vertical wind shear. This is not surprising since the more the wind turns with height, the more the gravity waves are absorbed. Therefore, the total turning angle determines much of the total WMF reduction. By $8 \mathrm{~km}$ height, most of the angle turning has been realized for W1-W6 (see $\Phi_{8 \mathrm{~km}}$ in Table 1).

In addition to the decay in magnitude with height, the WMF vector also changes its direction with height, as shown in Figs. $3 \mathrm{~b}$ and $3 \mathrm{e}$. The azimuth of the WMF increases with height for backing winds (Fig. 3b) but decreases with height for veering winds (Fig. 3e), indicating that the WMF vector turns with height in the same sense as the base-state wind. However, the WMF turns with height at a much smaller rate than the wind, which is in agreement with Shutts (1998). For example, the WMF vector rotates by less than $10^{\circ}$ from the surface to $8 \mathrm{~km}$ for W1 (thin dotted line in Fig. 3b), while the wind itself experiences a rotation of about $40^{\circ}$ (thick dotted line in Fig. 3b). Therefore, the WMF vector will be misaligned with the mean flow at high altitudes.

The magnitude of the WMF at the surface $\left|\mathbf{F}_{0}\right|$ is also of great importance since it determines the maximum wave momentum that can be deposited onto the mean flow. Figure 4a shows $\left|\mathbf{F}_{0}\right|$ as a function of $\Phi$ and inverse Ri. It is clear that $\left|\mathbf{F}_{0}\right|$ is symmetric about $\Phi=90^{\circ}$, and peaks at $\Phi=90^{\circ}$ for a given Ri, consistent with Fig. 3a; $\left|\mathbf{F}_{0}\right|$ increases with $\mathrm{Ri}$, but it is always smaller than in the constant flow case, also discussed in Grubišić and Smolarkiewicz (1997). The reduction of the surface WMF herein is more likely related to the linear type of vertical wind shear rather than the turning of the wind with height, because WMF values greater than that of constant flows have been obtained when taking into account wind curvature effects (Teixeira et al. 2004).

Misalignment of WMF with the basic wind at the surface has also been reported by Teixeira et al. (2004). Yet this is not always the case. Figure $4 \mathrm{~b}$ is akin to Fig. 4a except for the azimuth bias between $\mathbf{F}_{0}$ and $\mathbf{V}_{0}$, that is, $\left|\Psi_{0}-\psi_{0}\right|$. This azimuth bias, which vanishes at $\Phi=90^{\circ}$, is also symmetric with respect to $\Phi=90^{\circ}$, with two maxima near $\Phi=45^{\circ}$ and $135^{\circ} .\left|\Psi_{0}-\psi_{0}\right|$ decreases as the mean flow Richardson number increases. At Ri $=$ 0.25 , the maximum misalignment is about $17^{\circ}$ near $\Phi=$ $45^{\circ}$ and $135^{\circ}$. Figure $4 \mathrm{~b}$ reveals that for the WMF induced in directional shear flows, it may be misaligned with the ambient flow on the ground even when forced by an isotropic mountain, although the misalignment is generally no more than $17^{\circ}$.

\section{b. Vertical divergence of the wave momentum flux}

Figures $3 \mathrm{c}$ and $3 \mathrm{f}$ display the variation in the magnitude of the WMF divergence with height. Evidently, the vertical divergence of WMF vanishes in all cases at the surface, as analytically shown earlier. Unlike that of WMF, the magnitude of WMF divergence does not vary monotonously with height. Instead, it first increases with height, reaching a peak value at a certain altitude $z^{*}$ and then decreases with height again to near-zero values. This height variation of the WMF divergence is qualitatively similar to that of Shutts (1995). In addition, it appears that the WMF divergence would be greater for winds with larger values of $\Phi$ (Fig. 3c) and smaller values of Ri (larger vertical shear, Fig. 3f). However, it is not always the case, as can be seen below. 

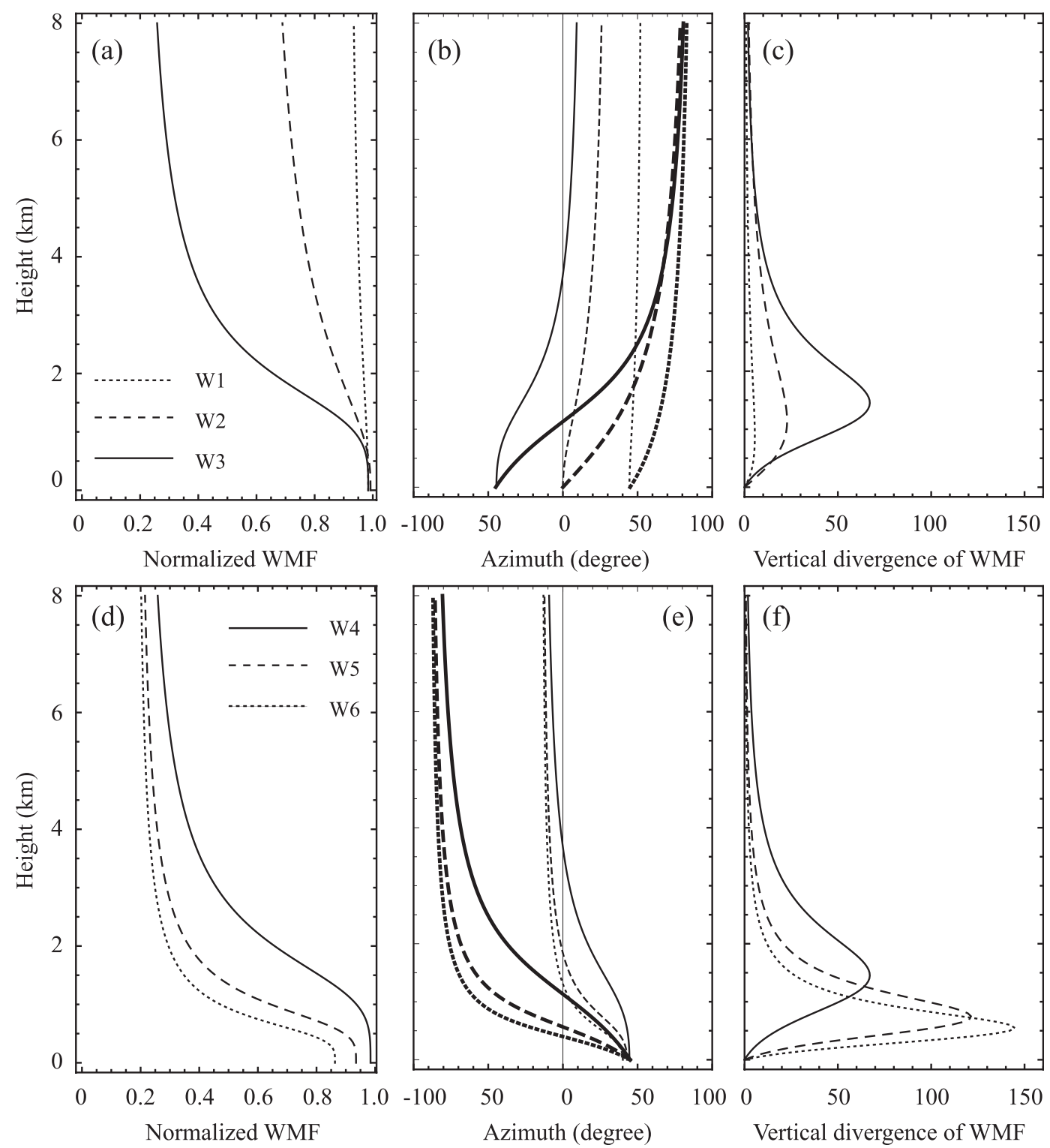

FIG. 3. Height variations of the (a),(d) magnitude and (b),(e) azimuth of the WMF and (c),(f) its vertical divergence for the wind profiles in Table 1. Thick lines in (b) and (e) correspond to the azimuths of the base-state winds. The magnitude of the WMF is normalized with respect to Eq. (28); i.e., the analytical value of WMF of a constant flow having the same surface wind speed.

From Eq. (26), the vertical derivative of $|\mathbf{L}(z)|$ is given by

$$
\frac{d|\mathbf{L}(z)|}{d z}= \pm 0.5 \bar{\rho} N a h_{0}^{2} \frac{\left|\mathbf{V}_{z}\right|^{2}}{\left|\mathbf{V}_{0}\right|} \frac{Q(\delta, \Phi, \mathrm{Ri}) \sin (\delta)}{\sin ^{2}\left[\Phi \sqrt{1-\sin ^{2} \delta / 4 \mathrm{Ri}}\right]},
$$

where

$$
\delta(z)=\left|\psi(z)-\chi_{0}\right|=\Phi-\left|\psi(z)-\psi_{0}\right|
$$

$$
\begin{aligned}
Q(\delta, \Phi, \mathrm{Ri})= & \sin (2 \delta-\Phi)\left[1-\frac{\sin ^{2}(\delta)}{4 \mathrm{Ri}}\right] \\
& +\sin (\delta-\Phi) \cos \delta\left[1-\frac{\sin ^{2}(\delta)}{2 \mathrm{Ri}}\right]
\end{aligned}
$$

It is feasible to write $\mathbf{L}(z)$ as $\mathbf{L}[\delta(z)]$ since $\delta(z)$ is a singlevalued monotonic function of $z$. According to Eq. (30), the maximum WMF divergence, that is $\left|\mathbf{L}\left(z^{*}\right)\right|$, appears at "height" $\delta_{0}$, where $Q\left(\delta_{0}, \Phi, \mathrm{Ri}\right)=0$. Unfortunately, it is hard to obtain the analytic solution for $Q$ to Eq. (30), so we solve it using numerical integration. 

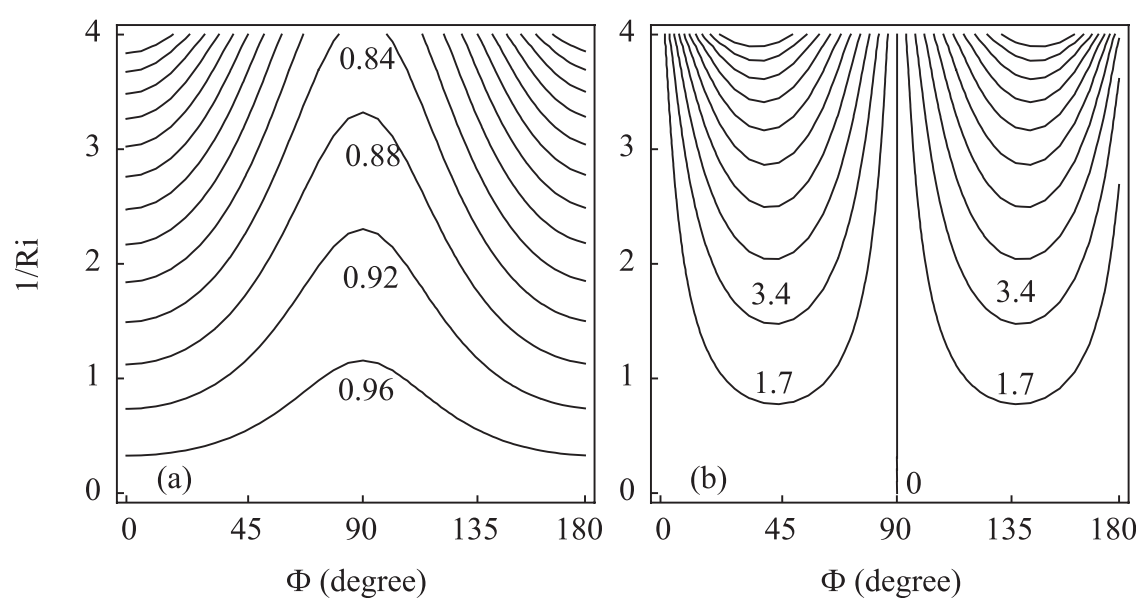

FIG. 4. Contour plots of (a) the magnitude of normalized WMF at the surface and (b) the azimuth difference between surface wind and surface WMF as a function of $\Phi$ and inverse Ri. Intervals are (a) $0.04^{\circ}$ and (b) $1.7^{\circ}$.

Figures $5 \mathrm{a}$ and $5 \mathrm{~b}$ are the counterplots of $Q$ as a function of $\Phi$ and $\delta$ for fixed $\mathrm{Ri}=4$ and 0.5 , respectively. In each case, there are two zero-value contours of $Q(\delta, \Phi)=0$ (dashed lines). Nevertheless, only the upper one (thick dashed line) is physically feasible, since $\delta$ should be smaller than $\Phi$ from Eq. (31). This upper solution curve is roughly asymptotic at large $\Phi$ to the straight line given by

$$
\delta=\Phi / 2
$$

Substituting Eq. (33) along with Eq. (29) into the expressions of the wind profile and WMF divergence, that is, Eqs. (11) and (26), one can obtain estimates for the altitude and magnitude of the maximum WMF divergence, as shown:

$$
\begin{gathered}
z_{\max }=\left|\mathbf{V}_{0}\right| /\left|\mathbf{V}_{z}\right| \\
\left|\mathbf{L}\left(z_{\max }\right)\right|=\frac{\bar{\rho} a h_{0}^{2} N^{2}}{4} \tan \frac{\Phi}{2} \sqrt{1-\left(1-\frac{1}{2 \mathrm{Ri}} \sin ^{2} \frac{\Phi}{2}\right)^{2}}
\end{gathered}
$$

It should be emphasized again that $z_{\max }$ and $\left|\mathbf{L}\left(z_{\max }\right)\right|$ are only estimates for their true value because Eq. (33) is not the exact solution to $d|\mathbf{L}(z)| / d z=0$.

From Eq. (34), $z_{\max }$ is independent of the maximum turning angle of the wind with height. However, the peak WMF divergence indeed occurs at different heights for winds with various $\Phi$ (see Fig. 3c). Figure 6 shows the altitude of the maximum WMF divergence obtained numerically from Eq. (26), which is considered
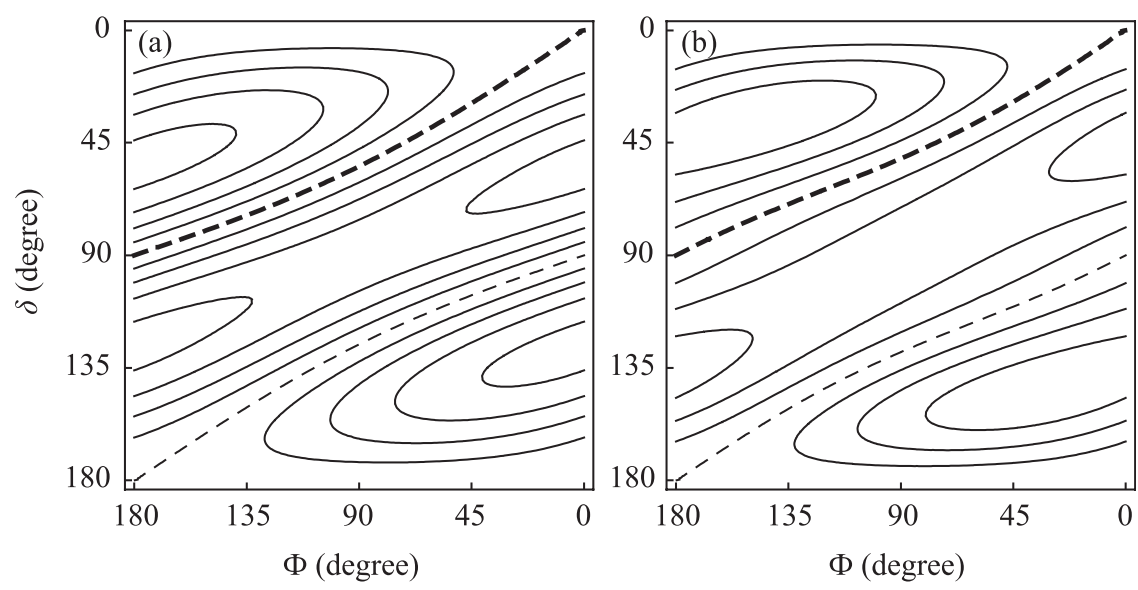

FIG. 5. Contour plots of $Q(\delta, \Phi)$ as a function of $\Phi$ and $\delta$ for (a) $\mathrm{Ri}=4$ and (b) $\mathrm{Ri}=0.5$. Dashed lines denote zero-value contours. 


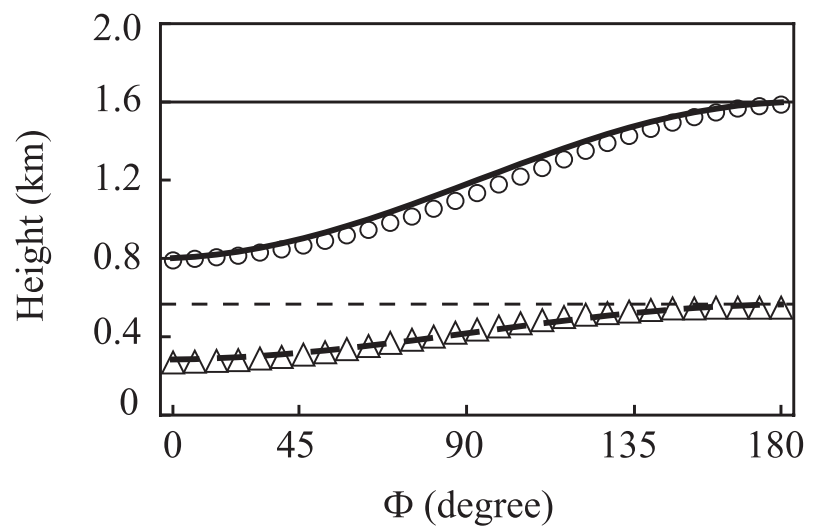

FIG. 6. Altitudes of the maximum WMF divergence for fixed $\mathrm{Ri}=4$ (solid lines and circles) and $\mathrm{Ri}=0.5$ (dashed lines and triangles). Symbols represent the true value obtained from Eq. (26). Thin lines and thick lines are estimates given by Eqs. (34) and (36), respectively.

as the true value of $z^{*}$. It is apparent that $z^{*}$ is asymptotic to $z_{\max }$ at $\Phi=180^{\circ}$, yet decreases gradually as $\Phi$ decreases, eventually halved at $\Phi=0^{\circ}$. This actual variation of $z^{*}$ with $\Phi$ shown in Fig. 6 suggests a modified empirical form of equation

$$
Z_{\max }=0.25(3-\cos \Phi) z_{\max }=0.25(3-\cos \Phi)\left|\mathbf{V}_{0}\right| /\left|\mathbf{V}_{z}\right|
$$

to account for the influence of $\Phi$. Plotted in Fig. 6, the $Z_{\max }$ given by the equation agrees with $z^{*}$ very well, even at $\mathrm{Ri}$ as low as 0.5 (see thick solid and thick dashed lines).

Despite the discrepancy between $z_{\max }$ and $z^{*},\left|\mathbf{L}\left(z_{\max }\right)\right|$ still provides an accurate estimate for $\left|\mathbf{L}\left(z^{*}\right)\right|$, as shown in Fig. 7. The simplicity and computational ease of Eqs. (35) and (36) make them very attractive. From Eq. (36), the altitude corresponding to the maximum WMF divergence is proportional to the ratio of surface wind speed to vertical wind shear intensity, and it increases as the maximum wind turning angle increases. Taking derivative of Eq. (35) with respect to $\Phi$ yields

$$
\begin{aligned}
\frac{d\left|\mathbf{L}\left(z_{\max }\right)\right|}{d \Phi}= & \frac{2 \bar{\rho} a h_{0}^{2}\left|\mathbf{V}_{z}\right|^{2} \tan ^{2}(\Phi / 2)}{\sqrt{1-\left[1-\sin ^{2}(\Phi / 2) /(2 \mathrm{Ri})\right]^{2}}} \\
& \times\left\{\sin ^{2} \frac{\Phi}{2}\left(1-\frac{1}{4 \mathrm{Ri}}\right)\right. \\
& \left.+2 \cos ^{2} \frac{\Phi}{2}\left[1-\frac{\sin ^{2}(\Phi / 2)}{4 \mathrm{Ri}}\right]\right\},
\end{aligned}
$$

which is always positive for any $\mathrm{Ri}$ larger than 0.25 . Therefore, $\left|\mathbf{L}\left(z_{\max }\right)\right|$ increases with $\Phi$, consistent with that of $\left|\mathbf{L}\left(z^{*}\right)\right|$ (see symbols in Fig. 7a). Similarly, by differentiating Eq. (35) with respect to $\mathrm{Ri}$, the greatest $\left|\mathbf{L}\left(z_{\max }\right)\right|$ for a given $\Phi$ is at

$$
\mathrm{Ri}_{\text {max }}=0.25(1-\cos \Phi) .
$$

For $\Phi<90^{\circ}, \mathrm{Ri}_{\max }$ falls below 0.25 , indicating an overall decrease of the peak WMF divergence with Ri (see triangles and dashed line in Fig. 7b). On the contrary when $\Phi>90^{\circ}, \mathrm{Ri}_{\text {max }}$ is greater than 0.25 , such that the peak WMF divergence first increases and then decreases with $\mathrm{Ri}$ (see circles and solid line in Fig. 7b). However, the increase in the peak WMF divergence with $\mathrm{Ri}$ is restricted within a narrow range of $\mathrm{Ri}$ because $\mathrm{Ri}_{\text {max }}$ is no more than 0.5 even in the extreme limit of $\Phi=180^{\circ}$. Therefore, the magnitude of the peak WMF divergence will almost always decrease as the ambient flow Richardson number increases.
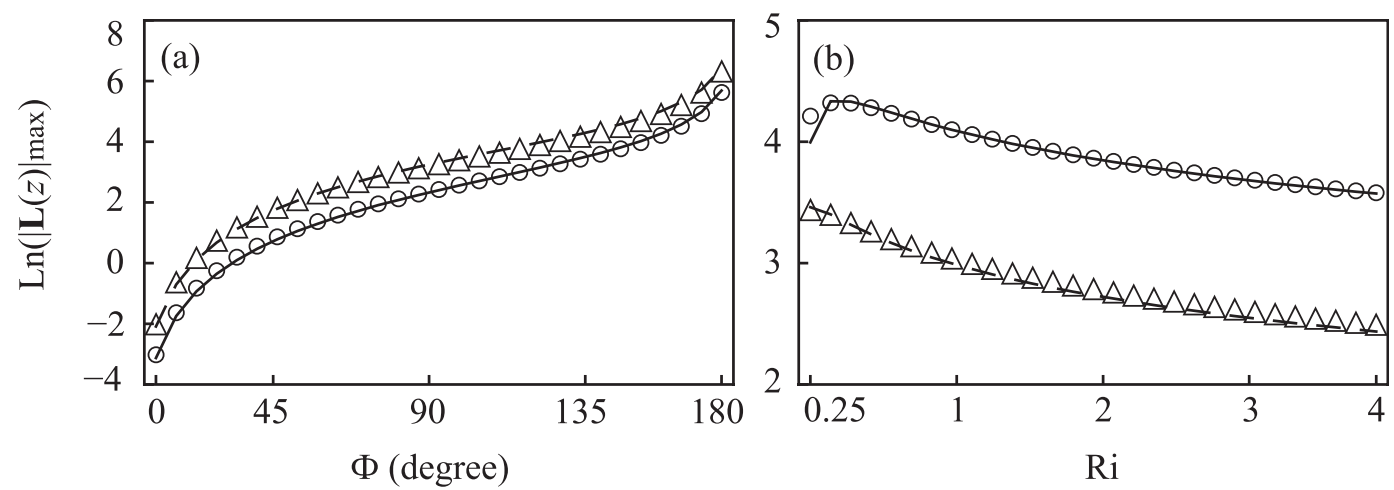

FIG. 7. Magnitude of the maximum WMF divergence $\left(\mathrm{kg} \mathrm{m}^{-2} \mathrm{~s}^{-2}\right)$ as a function of (a) $\Phi$ and (b) Ri. Symbols represent the true value obtained from Eq. (26); lines denote estimates given by Eq. (35). Solid (dashed) line and circles (triangles) in (a) are for $\mathrm{Ri}=4(0.5)$, while they are for $\Phi=135^{\circ}\left(45^{\circ}\right)$ in (b). 


\section{Further discussion}

The presence of the directional wind shear places constraints on the vertical propagation of gravity waves, such that the wave momentum might be trapped in the lower atmosphere. While in unidirectional flows, gravity waves are capable of freely transporting the wave momentum upward until a single critical level is encountered or when wave breaking takes place in the upper atmosphere due to the density effect on wave amplitude (Lindzen 1981).

According to linear theory, gravity waves in unidirectional flows deposit wave momentum onto the airflow at a single level. Mathematically, there will be an exceedingly large vertical divergence of the WMF at that level. Under this situation, the influence of gravity waves on the mean flow is difficult to represent in numerical models and ought to be parameterized (Palmer et al. 1986; Scinocca and McFarlane 2000; Kim et al. 2003). In contrast, the wave momentum produced in directional shear flows is continuously deposited onto the mean flow over a deep layer. The vertical divergence of the WMF tends to be smaller and can be better represented by numerical models with sufficient horizontal (and reasonably high vertical) resolutions. This may be an important difference for the numerical weather prediction purposes.

The formula of the WMF divergence obtained here is exact (to the extent that the problem is nearly linear) and can be readily implemented in the governing equations of numerical models. However, in reality, the height variation of the ambient air density (Smith 1979) and anisotropy of mountains (Gregory et al. 1998) should be taken into account. The assumption of hydrostatic waves also requires careful consideration. As studied by Keller (1994), in case of strongly sheared flows, the hydrostatic assumption may not be justified even for relatively large-scale topographic forcing. The nonhydrostatic effect can lead to a downstream shift of the WMF. Moreover, by neglecting the rotation of the Earth, inertial gravity waves (e.g., Jones 1967; Shen and Lin 1999; Shutts 2003) are a priori excluded, which may play an important role in the geostrophic adjustment process (Holton 2004).

This study is based on linear wave theory, such that it is least valid for large-amplitude gravity waves. Some weather phenomena related to nonlinear waves-for instance, severe downslope windstorms (Smith 1985), lee vortices (Smolarkiewicz and Rotunno 1989), and lee vortex shedding (Schär and Durran 1997)—can modify the WMF significantly (Miranda and James 1992; Eckermann et al. 2010). The wave momentum transport by nonlinear mountain waves generated in directionally sheared flows will be investigated in the future, primarily through numerical means.

\section{Conclusions}

Using linear, hydrostatic mountain wave theory, general expressions are derived for the wave momentum flux (WMF) and its vertical divergence induced in directional shear flows over three-dimensional obstacles. The inviscid ambient flow is horizontally uniform and varies linearly with height, such that the wind profile curvature effect is neglected. The rotation of the Earth, diabatic heating, and surface friction are also omitted. Properties of the WMF and its vertical divergence are studied by applying the deduced formulas to a specific, circular bell-shaped mountain for several idealized wind profiles. Major results are summarized as follows.

In the presence of directional wind shear, there exist an infinite number of critical levels at different heights. Terrain-forced gravity waves are thus continuously absorbed by the mean flow during their upward propagation. As a consequence of this directional shear critical layer absorption, the WMF decreases in magnitude with height. The maximum turning angle of the basestate wind with height $(\Phi)$ is found playing a crucial role in causing the decay of WMF with height. The wave momentum can be freely transported to the upper atmosphere with very little attenuation when the basic flow turns with height by fewer degrees. On the contrary, for flows turning substantially with height, the wave momentum is prone to being trapped in the lower atmosphere. Moreover, the WMF vector also rotates with height in the same sense as the background wind but at a much slower rate, thus becoming misaligned with the wind at high altitudes.

The vertical divergence of WMF is found to always vanish at the surface. Aloft, it points perpendicularly to the left (right) of the mean flow that backs (veers) with height. The magnitude of the WMF divergence first increases with height until reaching its maximum value. Thence, it begins to decrease with height, toward zero value. The altitude where the WMF divergence peaks increases as the maximum wind turning angle increases. It is proportional to the wind speed at the surface but inversely proportional to the vertical wind shear intensity. The magnitude of the maximum WMF divergence also shows an increase with the maximum wind turning angle, whereas it in general decreases as the environmental flow Richardson number (Ri) increases.

The WMF at the surface was also studied. It increases as the ambient flow Richardson number increases. However, it is always smaller than that in the constant flow case due to the linear vertical shear. For a given $\mathrm{Ri}$, 
the surface WMF peaks at $\Phi=90^{\circ}$. Only in this situation is the WMF aligned with the wind at the surface. The maximum misalignment between the azimuths of surface WMF and surface wind, which decreases as the Ri increases, appears at about $\Phi=45^{\circ}$ and $135^{\circ}$.

Gravity waves that develop in directionally sheared flows are common in the real atmosphere. For example, in a case of cold-air outbreak, northerly flows at the low levels will back with height, eventually becoming westerlies at the upper levels. Orographic gravity waves are expected to form when the northerly flow encounters a mountain and will propagate upward in a directionally sheared flow. We believe the results of this study have important implications on gravity wave drag parameterization in coarser-resolution numerical models and on the possible explicit representation of mountain gravity wave in high-resolution numerical models, and improving our understanding of such waves in the real world and in numerical models. Although the gravity wave drag parameterization associated with the wave momentum flux divergence in directionally sheared flows may be different from the drag due to the breaking of finite amplitude waves below critical levels, that is the subject of commonly used gravity wave drag parameterization schemes. Further discussions on some of these points are found in section 5 .

Acknowledgments. This work was primarily supported by National Science Foundation of China Grants 40775034, 40575017, and 40830958, and the National Grand Fundamental Research 973 Program of China (2009CB421502). Xin Xu was supported by the Chinese Scholarship Council for his extended visit at the Center for Analysis and Prediction of Storms, University of Oklahoma. The third author acknowledges the support of the National Thousand Person Plan hosted at Nanjing University and the support of NSF Grants AGS-0802888, OCI-0905040, AGS-0941491, AGS-1046171, and AGS1046081.

\section{REFERENCES}

Booker, J. R., and F. P. Bretherton, 1967: The critical layer for internal gravity waves in a shear flow. J. Fluid Mech., 27, 513539.

Bretherton, F. P., 1966: The propagation of groups of internal gravity waves in a shear flow. Quart. J. Roy. Meteor. Soc., 92, 466-480.

_ 1969: Momentum transport by gravity waves. Quart. J. Roy. Meteor. Soc., 95, 213-243.

Broad, A. S., 1995: Linear theory of momentum fluxes in 3-D flows with turning of the mean wind with height. Quart. J. Roy. Meteor. Soc., 121, 1891-1902.

Crapper, G. D., 1959: A three-dimensional solution for waves in the lee of mountains. J. Fluid Mech., 6, 51-76.
Doyle, J. D., and Q. Jiang, 2006: Observations and numerical simulations of mountain waves in the presence of directional wind shear. Quart. J. Roy. Meteor. Soc., 132, 1877-1905.

Eckermann, S. D., J. Lindeman, D. Broutman, J. Ma, and Z. Boybeyi, 2010: Momentum fluxes of gravity waves generated by variable Froude number flow over three-dimensional obstacles. J. Atmos. Sci., 67, 2260-2278.

Eliassen, A., and E. Palm, 1961: On the transfer of energy in stationary mountain waves. Geofys. Publ., 22, 1-23.

Fritts, D. C., 1984: Gravity wave saturation in the middle atmosphere: A review of theory and observations. Rev. Geophys., 22, 275-308.

_ , and T. J. Dunkerton, 1984: A quasi-linear study of gravity-wave saturation and self-acceleration. J. Atmos. Sci., 41, 3272-3289.

Gregory, D., G. J. Shutts, and J. R. Mitchell, 1998: A new gravitywave-drag scheme incorporating anisotropic orography and low-level wave breaking: Impact upon the climate of the UK Meteorological Office Unified Model. Quart. J. Roy. Meteor. Soc., 124, 463-493.

Grubišić, V., and P. K. Smolarkiewicz, 1997: The effect of critical levels on 3D orographic flows: Linear regime. J. Atmos. Sci., 54, 1943-1960.

Holton, J. R., 1983: The influence of gravity wave breaking on the general circulation of the middle atmosphere. J. Atmos. Sci., 40, 2497-2507.

—, 2004: An Introduction to Dynamic Meteorology. 4th ed. Academic Press, 535 pp.

Jones, W. L., 1967: Propagation of internal gravity waves in fluids with shear flow and rotation. J. Fluid Mech., 30, 439-448.

Keller, T. L., 1994: Implications of the hydrostatic assumption on atmospheric gravity waves. J. Atmos. Sci., 51, 1915-1929.

Kim, Y. J., S. D. Eckermann, and H. Y. Chun, 2003: An overview of the past, present and future of gravity-wave drag parametrization for numerical climate and weather prediction models. Atmos.-Ocean, 41, 65-98.

Lin, Y.-L., 2007: Mesoscale Dynamics. Cambridge University Press, 630 pp.

Lindzen, R. S., 1981: Turbulence and stress owing to gravity wave and tidal breakdown. J. Geophys. Res., 86 (C10), 9707-9714.

Lott, F., 1999: Alleviation of the stationary biases in a GCM through a mountain drag parameterization scheme and a simple representation of mountain lift forces. Mon. Wea. Rev., 127, 788-801.

Martin, A., and F. Lott, 2007: Synoptic responses to mountain gravity waves encountering directional critical levels. J. Atmos. Sci., 64, 828-848.

McFarlane, N. A., 1987: The effect of orographically excited gravity wave drag on the general circulation of the lower stratosphere and troposphere. J. Atmos. Sci., 44, 1775-1800.

Miranda, P. M. A., and I. N. James, 1992: Non-linear threedimensional effects on gravity-wave drag: Splitting flow and breaking waves. Quart. J. Roy. Meteor. Soc., 118, 1057-1081.

Palmer, T. N., G. J. Shutts, and R. Swinbank, 1986: Alleviation of systematic westerly bias in general circulation and numerical weather prediction models through an orographic gravity wave drag parameterization. Quart. J. Roy. Meteor. Soc., 112, 1001-1039.

Phillips, D. S., 1984: Analytical surface pressure and drag for linear hydrostatic flow over three-dimensional elliptical mountains. J. Atmos. Sci., 41, 1073-1084.

Schär, C., and D. R. Durran, 1997: Vortex formation and vortex shedding in continuously stratified flows past isolated topography. J. Atmos. Sci., 54, 534-554. 
Scinocca, J. F., and N. A. McFarlane, 2000: The parametrization of drag induced by stratified flow over anisotropic orography. Quart. J. Roy. Meteor. Soc., 126, 2353-2393.

— eterization of orographic gravity wave drag. J. Atmos. Sci., 67, 2537-2546.

Shen, B.-W., and Y.-L. Lin, 1999: Effects of critical levels on twodimensional back-sheared flow over an isolated mountain ridge on an $f$ plane. J. Atmos. Sci., 56, 3286-3302.

Shutts, G. J., 1995: Gravity-wave drag parametrization over complex terrain: The effect of critical level absorption in directional wind-shear. Quart. J. Roy. Meteor. Soc., 121, 1005-1021. , 1998: Stationary gravity-wave structure in flows with directional wind shear. Quart. J. Roy. Meteor. Soc., 124, 14211442.

- 2003: Inertial-gravity wave and neutral Eady wave trains forced by directionally sheared flow over isolated hills. J. Atmos. Sci., 60, 593-606.

, and A. Gadian, 1999: Numerical simulations of orographic gravity waves in flows which back with height. Quart. J. Roy. Meteor. Soc., 125, 2743-2765.
Smith, R. B., 1979: Some aspects of the quasi-geostrophic flow over mountains. J. Atmos. Sci., 36, 2385-2393.

_ 1980: Linear theory of stratified hydrostatic flow past an isolated mountain. Tellus, 32, 348-364.

_ 1985: On severe downslope winds. J. Atmos. Sci., 42, 25972603.

Smolarkiewicz, P. K., and R. Rotunno, 1989: Low Froude number flow past three-dimensional obstacles. Part I: Baroclinically generated lee vortices. J. Atmos. Sci., 46, 1154-1164.

Teixeira, M. A. C., and P. M. A. Miranda, 2006: A linear model of gravity wave drag for hydrostatic sheared flow over elliptical mountains. Quart. J. Roy. Meteor. Soc., 132, 2439-2458.

— , and — 2009: On the momentum fluxes associated with mountain waves in directionally sheared flows. J. Atmos. Sci., 66, 3419-3433.

—_ - and M. A. Valente, 2004: An analytical model of mountain wave drag for wind profiles with shear and curvature. J. Atmos. Sci., 61, 1040-1054.

,-- J. L. Argain, and M. A. Valente, 2005: Resonant gravity-wave drag enhancement in linear stratified flow over mountains. Quart. J. Roy. Meteor. Soc., 131, 1795-1814. 